\title{
Measuring the Primordial Deuterium Abundance during the Cosmic Dark Ages
}

\author{
Kris Sigurdson* and Steven R. Furlanetto ${ }^{\dagger}$ \\ California Institute of Technology, Mail Code 130-33, Pasadena, California 91125, USA
}

(Received 10 May 2005; published 29 August 2006)

\begin{abstract}
We discuss how measurements of fluctuations in the absorption of cosmic microwave background photons by neutral gas at redshifts $z \approx 7-200$ could reveal the primordial deuterium abundance of the Universe. The strength of the cross-correlation of brightness-temperature fluctuations in the redshifted $21-\mathrm{cm}$ line of hydrogen with those in the redshifted $92-\mathrm{cm}$ line of deuterium is proportional to the value of the deuterium-to-hydrogen ratio $[\mathrm{D} / \mathrm{H}]$ fixed during big bang nucleosynthesis. Although challenging, this measurement would provide the cleanest possible determination of $[\mathrm{D} / \mathrm{H}]$, free from contamination by structure formation processes at lower redshifts. We additionally report our result for the thermal spinchange cross section in deuterium-hydrogen scattering.
\end{abstract}

DOI: 10.1103/PhysRevLett.97.091301

Introduction. - After the cosmic microwave background $(\mathrm{CMB})$ radiation decoupled from the baryons at a redshift $z \approx 1100$, most $\mathrm{CMB}$ photons propagated unfettered through the neutral primordial medium. This has allowed exquisite measurements of the temperature fluctuations in the primordial plasma at the surface of last scattering, and the statistical properties of these fluctuations have recently been used, in conjunction with other observations, to determine the cosmology of our Universe [1]. After the photons kinetically decoupled from the gas at $z \sim 200$, the latter cooled adiabatically with $T_{g} \propto(1+z)^{2}$, faster than the $T_{\gamma} \propto(1+z)$ cooling of the CMB. This epoch, with most of the baryons in the form of relatively cold neutral atoms and before the first stars formed, is known as the cosmic dark ages.

The reason most CMB photons propagate unimpeded through the neutral primordial gas is elementary quantum mechanics - atoms absorb nonionizing radiation only at the discrete wavelengths determined by the differences of their atomic energy levels. One interesting example is the well-known 21-cm spin-flip transition [2], due to the hyperfine splitting of the ground state of the hydrogen $(\mathrm{H})$ atom. At any given $z, \mathrm{CMB}$ photons with wavelength $\lambda_{21}=21.1 \mathrm{~cm}$ can resonantly excite this transition. By measuring brightness-temperature fluctuations due to density fluctuations in the neutral gas [3], radio telescopes observing at $\lambda=(1+z) \lambda_{21}$ can probe the matter power spectrum at $z \approx 30-200$ [4].

In this Letter, we discuss another application of these measurements. Less well-known than the $21-\mathrm{cm}$ transition of neutral $\mathrm{H}$ is the spin-flip transition of neutral deuterium (D) at $\lambda_{92}=91.6 \mathrm{~cm}$ [5]. We show below that crosscorrelating brightness-temperature fluctuations at a wavelength $\lambda_{\mathrm{H}}=(1+z) \lambda_{21}$ with those at a wavelength $\lambda_{\mathrm{D}}=$ $(1+z) \lambda_{92}$ allows a measurement of the primordial D abundance. In principle, this technique could constrain the primordial value of $[\mathrm{D} / \mathrm{H}] \equiv n_{\mathrm{D}} / n_{\mathrm{H}}$ to better than $1 \%$. While there is no physical obstacle to such a measurement, it would certainly be technically challenging and require a heroic experimental effort; simply confirming
PACS numbers: 98.80.Ft, 32.10.Fn, 34.50.-s, 95.30.Dr

the presence of $\mathrm{D}$ via cross-correlation during the cosmic dark ages is a significantly easier goal.

Deuterium has long been recognized as our best "baryometer" because its primeval relic abundance is so sensitive to the baryon-to-photon ratio $\eta=n_{b} / s$. Moreover, big bang nucleosynthesis (BBN) [6] is the only known natural production mechanism, although mechanisms inside galaxies can destroy it [7]. The measurement we describe below could thus determine the true BBN abundance of $\mathrm{D}$ and, in principle, might improve $\mathrm{BBN}$ constraints to the baryon density of the Universe $\Omega_{b} h^{2}$.

Hyperfine structure of $H$ and $D$ atoms. -The $\boldsymbol{\mu} \cdot \mathbf{B}$ interaction between the magnetic moments of the electron and the nucleus splits the ground state of single-electron atoms into eigenstates of the total spin operator $\mathbf{F}=\mathbf{S}+\mathbf{I}$ with eigenvalues $F_{+}=I+1 / 2$ and $F_{-}=I-1 / 2$ and $\Delta E=(16 / 3) F_{+} \mu_{B}\left(g_{N} \mu_{N} / a_{0}^{3}\right)$ (e.g., [8]). Here $\mathbf{S}$ is electron spin, $\mathbf{I}$ is nuclear spin, $a_{0}$ is the Bohr radius, $\mu_{B}$ is the Bohr magneton, $\mu_{N}$ is the nuclear magneton, and $g_{N}$ is the nuclear $g$ factor $\left(g_{p}=5.56\right.$ for $\mathrm{H} ; g_{\mathrm{D}}=0.857$ for D). The proton, with $I=1 / 2$, splits the $\mathrm{H}$ ground state into a triplet with $F_{+}=1$ and a singlet with $F_{-}=0$. The deuteron, with $I=1$, splits the $\mathrm{D}$ ground state into a quartet with $F_{+}=3 / 2$ and a doublet with $F_{-}=1 / 2$.

The population of atoms in the excited spin state relative to the ground state $n_{+} / n_{-}=\left(g_{+} / g_{-}\right) \exp \left\{-T_{\star} / T_{s}\right\}$ can be characterized by a spin temperature $T_{s}$. Here $g_{+}=$ $2 F_{+}+1$ and $g_{-}=2 F_{-}+1$ are spin degeneracy factors and $T_{\star}=\Delta E / k_{B}$. For $\mathrm{H}$ and $\mathrm{D}$, we have $T_{\star}^{\mathrm{H}}=0.0682 \mathrm{~K}$, $T_{\star}^{\mathrm{D}}=0.0157 \mathrm{~K},\left(g_{+}^{\mathrm{H}} / g_{-}^{\mathrm{H}}\right)=3$, and $\left(g_{+}^{\mathrm{D}} / g_{-}^{\mathrm{D}}\right)=2$.

Three factors determine $T_{s}$ : absorption of 21-cm CMB photons, absorption and reemission of Lyman- $\alpha$ photons [the Wouthuysen-Field (WF) effect $[9,10]]$, and atomic spin-change collisions (free electrons are unimportant in these environments [10]). The first drives $T_{s}$ toward $T_{\gamma}$, while the latter two drive it toward the gas temperature $T_{g}$. In equilibrium, the spin temperature of a species $X$ is $T_{s}^{X}=$ $\left(1+\chi^{X}\right) T_{g} T_{\gamma} /\left(T_{g}+\chi^{X} T_{\gamma}\right)$, where $\chi^{X} \equiv \chi_{c}^{X}+\chi_{\alpha}^{X}$ is the sum of the equilibrium threshold parameters for spinchange collisions and for radiative coupling through the 
WF effect. Explicitly, $\chi_{c}^{X}=\left(C_{+-}^{X} T_{\star}^{X}\right) /\left(A_{+-}^{X} T_{\gamma}\right)$ and $\chi_{\alpha}^{X}=$ $\left(P_{+-}^{X} T_{\star}^{X}\right) /\left(A_{+-}^{X} T_{\gamma}\right)$, where $C_{+-}^{X}$ is the collisional deexcitation rate, $A_{+-}^{X}$ is an Einstein coefficient, and $P_{+-}^{X} \propto P_{\alpha}$, where $P_{\alpha}$ is the total Lyman- $\alpha$ scattering rate. At $z \gg 10$, before the first galaxies formed, $P_{\alpha}$ is tiny and the WF effect can be neglected. However, it might have interesting consequences near $z \sim 10$.

$H$ - $H$ and $D-H$ collision rates. - While the cross section for $\mathrm{H}-\mathrm{H}$ spin-change collisions $\Sigma_{+-}^{\mathrm{HH}}$ is well-known [1113], we could not locate the D-H spin-change cross section for the temperatures of interest [14] and computed $\Sigma_{+-}^{\mathrm{DH}}$ using standard partial-wave phase-shift methods. Our results agree with Refs. [11-13] for $\Sigma_{+-}^{\mathrm{HH}}$, with Ref. [13] for $\Sigma_{+-}^{\mu \mathrm{H}}$, and with Ref. [16] for $\Sigma_{+-}^{\mathrm{DH}}$ at $1 \mathrm{~K}$ (after accounting for our more recent molecular potentials).

The collisional deexcitation rate is $C_{+-}^{X}=\bar{v}_{X \mathrm{H}} \bar{\Sigma}_{+-}^{X \mathrm{H}} n_{\mathrm{H}}$, where $\bar{v}_{X \mathrm{H}}=\sqrt{8 k_{B} T_{g} / \mu_{X \mathrm{H}} \pi}$ is the thermal velocity, $\bar{\Sigma}_{+-}^{X \mathrm{H}}$ is the thermally averaged spin-change cross section, and $n_{\mathrm{H}}$ is the number density of $\mathrm{H}$ atoms. In Fig. 1, we plot $\bar{\Sigma}_{+-}^{\mathrm{HH}}, \bar{\Sigma}_{+-}^{\mathrm{DH}}$, and $\bar{\Sigma}_{+-}^{\mu \mathrm{H}}$. While $\overline{\boldsymbol{\Sigma}}_{+-}^{\mathrm{HH}}$ falls off for $T_{g} \lesssim$ $100 \mathrm{~K}, \bar{\Sigma}_{+-}^{\mathrm{DH}}$ continues to rise to a peak near $T_{g} \sim 1 \mathrm{~K}$. This occurs because of low-energy $s$-wave and $p$-wave contributions to D-H scattering. These do not appear for the $\mathrm{H}-\mathrm{H}$ system due to its different reduced mass ( $\mu_{\mathrm{HH}} \approx$ $m_{\mathrm{H}} / 2$ while $\mu_{\mathrm{DH}} \approx 2 m_{\mathrm{H}} / 3$ ). The discussion of $\mathrm{D}-\mathrm{H}$ spin change in Ref. [10] did not account for this and incorrectly concluded that $\Sigma_{+-}^{\mathrm{DH}} \sim \Sigma_{+-}^{\mathrm{HH}}$.

Spin-temperature evolution. - In Fig. 2, we plot $T_{\gamma}, T_{g}$ (found using RECFAST [18]), $T_{s}^{\mathrm{H}}$, and $T_{s}^{\mathrm{D}}$ as a function of $z$. After the gas cools below $T_{\gamma}$, collisions keep $T_{s}^{\mathrm{H}}$ and $T_{s}^{\mathrm{D}}$ coupled to $T_{g}$. Near $z \sim 30$, collisions become inefficient for $\mathrm{H}$ and $T_{s}^{\mathrm{H}}$ returns to $T_{\gamma}$. $T_{s}^{\mathrm{D}}$ remains coupled to $T_{g}$ down

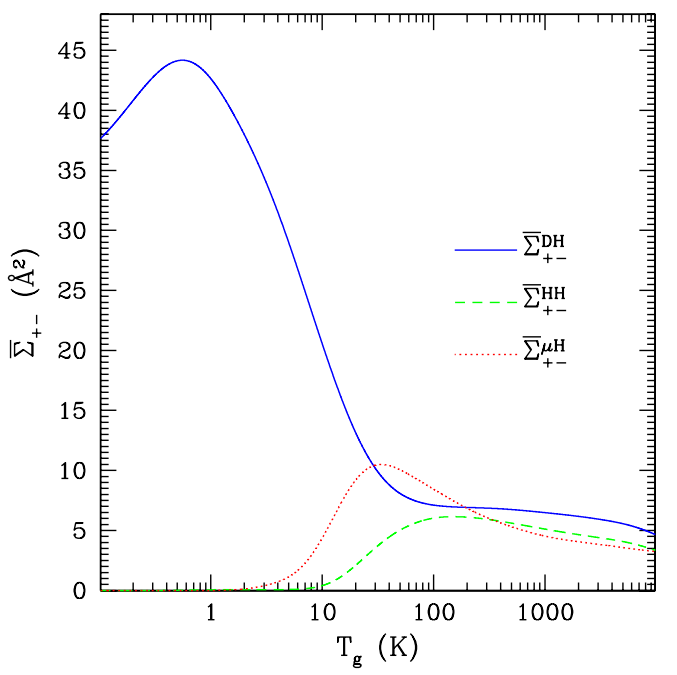

FIG. 1 (color online). The thermal spin-change cross sections that keep $T_{s}$ collisionally coupled to $T_{g}$ for D (solid line), $\mathrm{H}$ (dashed line), and muonium (dotted line). Although the potentials are identical, the peaks differ because of the reduced masses. to significantly lower redshift both because the lifetime of the excited state of $\mathrm{D}$ is relatively long $\left(A_{+-}^{\mathrm{H}} / A_{+-}^{\mathrm{D}}=\right.$ 61.35) and because $\bar{\Sigma}_{+-}^{\mathrm{DH}} \gg \bar{\Sigma}_{+-}^{\mathrm{HH}}$ at low $T_{g}$.

Brightness-temperature fluctuations. - When the spin temperature of a given species is less than $T_{\gamma}$, it will absorb CMB photons. The brightness temperature is $T_{b}^{X}=$ $a \tau_{X}\left(T_{s}^{X}-T_{\gamma}\right), \quad$ where $\tau_{X}=g_{+}^{X} c \lambda^{2} h A_{+-}^{X} n_{X} /\left[8\left(g_{+}^{X}+\right.\right.$ $\left.\left.g_{-}^{X}\right) \pi k_{B} T_{s}^{X} \mathcal{H}(z)\right]$ is the optical depth of the spin-flip transition in question, $a=1 /(1+z)$, and $\mathcal{H}(z)$ is the Hubble parameter. We are interested in correlations between brightness-temperature fluctuations $\delta T_{b}^{X}(\hat{\mathbf{n}}, a) \equiv$ $\beta^{X}(a) T_{b}^{X}(a) \delta(\hat{\mathbf{n}}, a)$ observed in a direction $\hat{\mathbf{n}}$ at wavelengths differing by a factor $\lambda_{92} / \lambda_{21}$. Here $\beta^{X}=1+$ $\chi_{\mathrm{c}}^{X} / \hat{\chi}^{X}+\Gamma\left\{T_{\gamma} /\left(T_{g}-T_{\gamma}\right)+\left(\chi_{c}^{X} / \hat{\chi}^{X}\right) d \ln \left(C_{+-}^{X}\right) / d \ln \left(T_{g}\right)\right\}$, $\delta(\hat{\mathbf{n}}, a)$ is the fractional density contrast, and $\hat{\chi}^{X} \equiv \chi^{X}(1+$ $\left.\chi^{X}\right)$. At high $z$, when $T_{g} \approx T_{\gamma}, \Gamma \rightarrow 0$ due to residual Thomson scattering with free electrons [19] (fluctuations are isothermal), but, as the gas begins to cool adiabatically, $\Gamma \rightarrow 2 / 3$ [20]. We have neglected the contributions to $\delta_{T_{b}}$ from fluctuations in the neutral fraction (likely to be small at high $z$ ) and, for simplicity, fluctuations in the gradient of the radial velocity $\delta_{\partial_{r} v_{r}}[20,21]$. The latter will enhance our signal by a factor of $\sim 1-2$. In Fig. 3 , we plot $T_{b}^{\mathrm{H}}, \tilde{T}_{b}^{\mathrm{D}}$, $\sigma_{T_{b}^{\mathrm{H}}}$, and $\tilde{\sigma}_{T_{b}^{\mathrm{D}}}\left(\right.$ where $T_{b}^{\mathrm{D}} \equiv \epsilon \tilde{T}_{b}^{\mathrm{D}}, \sigma_{T_{b}^{\mathrm{D}}} \equiv \epsilon \tilde{\sigma}_{T_{b}^{\mathrm{D}}}$, and $\epsilon \equiv$ $[\mathrm{D} / \mathrm{H}])$. We see that $\tilde{T}_{b}^{\mathrm{D}}$ and $\tilde{\sigma}_{T_{b}^{\mathrm{D}}}$ peak at much lower $z$ than their $\mathrm{H}$ counterparts because, as discussed above, $T_{s}^{\mathrm{D}}$ is coupled to $T_{g}$ to lower $z$. The lower set of curves in Fig. 3 have $P_{\alpha}=0$ throughout (appropriate for rapid late reionization), while the upper assume strong WF coupling and simultaneous heating at $z=14$ (appropriate if gradual reionization begins at about that time).

$D$ - $H$ cross-correlations. - We now calculate the crosscorrelation of brightness-temperature fluctuations across frequencies in the ratio $\lambda_{21}: \lambda_{92}$. We write the brightnesstemperature fluctuation due to $\mathrm{H}$ or $\mathrm{D}$ as $\mathrm{H}(\hat{\mathbf{n}}, a)=$ $\beta^{\mathrm{H}}(a) T_{b}^{\mathrm{H}}(a) \delta(\hat{\mathbf{n}}, a)$ and $\epsilon \mathrm{D}(\hat{\mathbf{n}}, a)=\epsilon \beta^{\mathrm{D}}(a) \tilde{T}_{b}^{\mathrm{D}}(a) \delta(\hat{\mathbf{n}}, a)$,

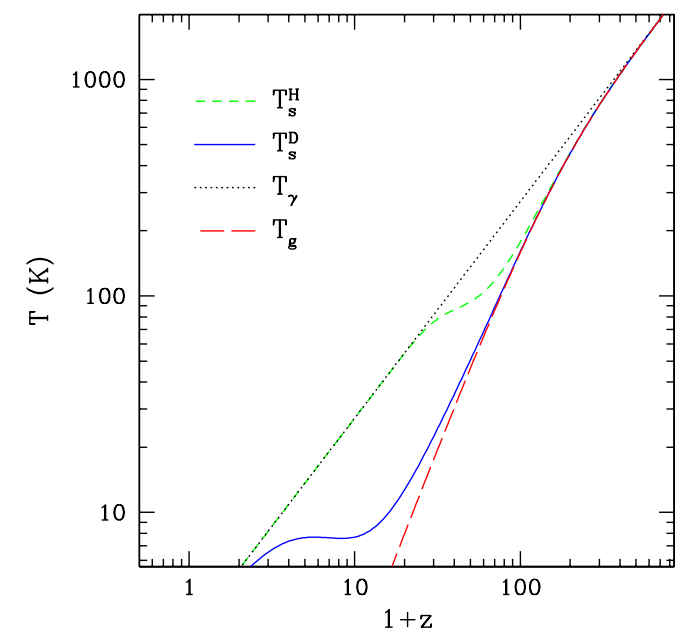

FIG. 2 (color online). The $\mathrm{H}$ and $\mathrm{D}$ spin temperatures as a function of $z$. Here we assume $P_{\alpha}=0$ for all $z$. 


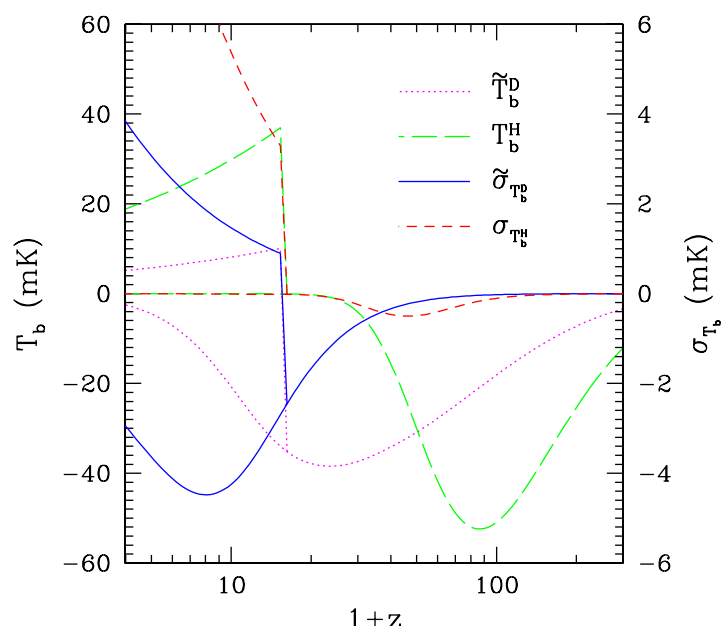

FIG. 3 (color online). The mean brightness temperatures $T_{b}^{\mathrm{H}}$ and $\tilde{T}_{b}^{\mathrm{D}}$ and the standard deviation of pixel-scale brightnesstemperature fluctuations $\sigma_{T_{b}^{\mathrm{H}}}$ and $\tilde{\sigma}_{T_{b}^{\mathrm{D}}}$ as a function of redshift. The $\sigma_{T_{b}}$ are shown for the benchmark experiment discussed in the text. The lower curves assume the gas remains cool, while the upper curves show the effect of heating the gas far above $T_{\gamma}$ at $z=14$.

respectively. A radio telescope observing at a frequency $\nu$ will measure the quantity $\mathcal{O}[\hat{\mathbf{n}} ; \nu]=\mathrm{H}\left(\hat{\mathbf{n}}, \nu / \nu_{21}\right)+$ $\epsilon \mathrm{D}\left(\hat{\mathbf{n}}, \nu / \nu_{92}\right)+N[\hat{\mathbf{n}} ; \nu]$, where $N[\hat{\mathbf{n}} ; \nu]$ is the noise. The product of observables at frequencies $\nu_{h}$ (the $\mathrm{H}$ band) and $\nu_{d} \equiv\left(\nu_{92} / \nu_{21}\right) \nu_{h}$ (the $\mathrm{D}$ band) is $\mathcal{O}\left[\hat{\mathbf{n}} ; \nu_{h}\right] \mathcal{O}\left[\hat{\mathbf{n}} ; \nu_{d}\right]=$ $\mathrm{H}_{h} \mathrm{H}_{d}+\epsilon \mathrm{H}_{h} \mathrm{D}_{d}+\mathrm{H}_{h} N_{d}+\epsilon \mathrm{D}_{h} \mathrm{H}_{d}+\epsilon^{2} \mathrm{D}_{h} \mathrm{D}_{d}+\epsilon \mathrm{D}_{h} N_{d}$, where we have introduced the shorthand $\mathrm{H}_{x} \equiv$ $\mathrm{H}\left(\hat{\mathbf{n}}, \nu_{x} / \nu_{21}\right), \quad \mathrm{D}_{x} \equiv \mathrm{D}\left(\hat{\mathbf{n}}, \nu_{x} / \nu_{92}\right), \quad$ and $\quad N_{x}=N\left[\hat{\mathbf{n}} ; \nu_{x}\right]$. Assuming that $\delta(\hat{\mathbf{n}}, a)$ is a zero-mean random field and uncorrelated noise, the expectation values (averaged over all the pixels observed on the sky) of most terms vanish as the $\nu_{h}$-band and $\nu_{d}$-band factors are uncorrelated. Only $\mathrm{H}_{h}$ and $\mathrm{D}_{d}$ are strongly correlated, and, thus, we have $\left\langle\mathcal{O}\left[\hat{\mathbf{n}} ; \nu_{h}\right] \mathcal{O}\left[\hat{\mathbf{n}} ; \nu_{d}\right]\right\rangle=\epsilon\left\langle\mathrm{H}_{h} \mathrm{D}_{d}\right\rangle$.

We now understand the crucial point of this Letter. The 21- and 92-cm fluctuations at these frequency separations must be correlated because they trace the same underlying patches of the Universe.

Note that we have neglected the relatively small intrinsic correlations $\left\langle\mathrm{H}_{h} \mathrm{H}_{d}\right\rangle$ that arise due to large-scale modes of the density field. These contribute $\leqslant 0.1 \%$ of the D crosscorrelation signal. If necessary, these correlations could be removed by correlating the fluctuations in the $\nu_{h}$ band with fluctuations at frequencies near but not equal to the corresponding $\nu_{d}$ band.

Signal estimate. - Our proposal is relatively straightforward: Brightness-temperature fluctuations in the same direction on the sky (the same angular pixel) at frequencies $\nu_{h}$ and $\nu_{d}=\left(\nu_{92} / \nu_{21}\right) \nu_{h}$ should be cross-correlated. At a given redshift $z=\nu_{21} / \nu_{h}-1=\nu_{92} / \nu_{d}-1$, the average signal contributed from a pixel in a frequency band $\Delta \nu_{h}$ about $\nu_{h}$ and $\Delta \nu_{d}=\left(\nu_{92} / \nu_{21}\right) \Delta \nu_{h}$ about $\nu_{d}$ is just the product of the standard deviations of the $\mathrm{H}$ and $\mathrm{D}$ brightness-temperature fluctuations: $\quad \epsilon\left\langle\mathrm{H}_{h} \mathrm{D}_{d}\right\rangle=$ $\epsilon \sigma_{\mathrm{H}_{h}} \tilde{\sigma}_{\mathrm{D}_{d}}=\epsilon \sigma_{\delta}^{2} \beta^{\mathrm{H}_{h}} T_{b}^{\mathrm{H}_{h}} \beta^{\mathrm{D}_{d}} \tilde{T}_{b}^{\mathrm{D}_{d}}$. Here $\sigma_{\delta}^{2}$ is the variance of density fluctuations averaged over the flat cylindrical volume of each pixel. Explicitly,

$$
\sigma_{\delta}^{2}=\frac{2}{\pi^{2}} \int_{0}^{\infty} d k k P(k) \int_{0}^{k} d k_{z} j_{0}^{2}\left(\xi k_{z} \rho\right) \frac{J_{1}^{2}\left(\sqrt{k^{2}-k_{z}^{2}} \rho\right)}{\left(k^{2}-k_{z}^{2}\right) \rho^{2}},
$$

for a pixel at redshift $z$ with comoving radius $\rho$ and thickness $2 \xi \rho$. The noise in each pixel is a combination in quadrature of the random noise $\left\langle N_{x}^{2}\right\rangle=\sigma_{N_{x}}^{2}$ and the confusion arising from the autocorrelation of the $\mathrm{H}$ and D fluctuations. In practice, since $\epsilon^{2}\left\langle\mathrm{D}_{x}^{2}\right\rangle \ll\left\langle\mathrm{H}_{x}^{2}\right\rangle$, we can always neglect the confusion due to $\mathrm{D}$ fluctuations in the noise. The average noise per pixel in the cross-correlation measurement is thus $\sqrt{\sigma_{\mathrm{H}_{h}}^{2}+\sigma_{N_{h}}^{2}} \sqrt{\sigma_{\mathrm{H}_{d}}^{2}+\sigma_{N_{d}}^{2}}$. Here $\sigma_{N_{x}}^{2}=T_{\text {sys }}^{2} /\left(f_{\text {cov }}^{2} \Delta \nu_{x} t_{\text {int }}\right) \quad$ (e.g., [22]), where $T_{\text {sys }} \simeq$ $6500\left[\nu_{\alpha} /(30 \mathrm{MHz})\right]^{-2} \mathrm{~K}$ is the noise temperature, $f_{\text {cov }}$ is the covering fraction of the array, and $t_{\text {int }}$ is the integration time. At redshift $z$, there are a total of $N_{\text {pix }}=16 f_{\text {sky }} /\left(\theta_{d}^{2}\right)$ pixels for an instrument with maximum baseline $L$ and angular resolution $\theta_{d}=\lambda_{d} / L=(1+z) \lambda_{92} / L$ in the $\mathrm{D}$ band that observes a fraction $f_{\text {sky }}$ of the sky. The signalto-noise contributed from the bands $\nu_{h}$ and $\nu_{d}$ at redshift $z$ is thus

$$
\frac{\mathcal{S}}{\mathcal{N}}(z)=\frac{\epsilon \sqrt{N_{\mathrm{pix}}} \sigma_{\mathrm{H}_{h}} \tilde{\sigma}_{\mathrm{D}_{d}}}{\sqrt{\sigma_{\mathrm{H}_{h}}^{2}+\sigma_{N_{h}}^{2}} \sqrt{\sigma_{\mathrm{H}_{d}}^{2}+\sigma_{N_{d}}^{2}}} .
$$

If $\mathcal{S} / \mathcal{N}(z)$ did not depend on $z$ and measurements were made over a total bandwidth $B_{h}$ in the $\mathrm{H}$ band [or $B_{d}=$ $\left(\nu_{92} / \nu_{21}\right) B_{h}$ in the $\mathrm{D}$ band], the total signal-to-noise ratio would just be Eq. (2) multiplied by a factor $\sqrt{B_{h} / \Delta \nu_{h}}$. In practice, as $\mathcal{S} / \mathcal{N}(z)$ varies with $z$, we calculate $(\mathcal{S} / \mathcal{N})_{\text {tot }}$ by summing $\mathcal{S} / \mathcal{N}(z)$ over all redshift bins in quadrature.

We note that our choice for $T_{\text {sys }}$ is only an estimate, and the noise (ultimately due to Galactic synchrotron radiation) varies strongly across the sky. Potential systematic effects including instrument calibration and foreground removal will undoubtedly make a detection more challenging and will necessitate the use of more sophisticated statistical estimators of the cross-correlation signal than the simple estimator we describe here. For instance, as we propose to cross-correlate brightness-temperature fluctuations that vary rapidly as a function of frequency, most foreground signals (which are expected to vary smoothly in frequency space) can be removed by applying a high-pass filter in cofrequency space to multiband observations (which would reject any signal smooth in frequency space) $[22,23]$. In fact, as we are looking for a cross-correlation signal, this type of measurement should be robust to even more pathological foreground and systematic effects. For example, a foreground or instrumental effect which leads to correlations between the $\nu_{h}$ band and the $\nu_{d}$ band may also lead to related correlations between the $\nu_{h}$ band and 
another frequency band $\nu_{o}$. However, a statistical estimator for $\epsilon=[\mathrm{D} / \mathrm{H}]$ can be devised that rejects correlations between the $\nu_{h}$ band and $\nu_{d}$ band if the fluctuations in these bands are also significantly correlated with the fluctuations in the $\nu_{o}$ band or any other band. The extent to which these or other techniques will be needed will become clearer in the years to come as $21-\mathrm{cm}$ experiments gain real-world experience with these issues.

If collisions dominate the coupling between $T_{s}^{\mathrm{H}}$ and $T_{g}$ down to $z \sim 7$, then we estimate that a value $[\mathrm{D} / \mathrm{H}] \sim 3 \times$ $10^{-5}$ could be detected at $1-2 \sigma$ by a benchmark experiment with $L \sim 7.5 \mathrm{~km}$ and $\Delta \nu_{d} \sim 100 \mathrm{kHz}$ in $\sim 6$ years. If, however, the first generation of stars created a flux of Lyman- $\alpha$ photons which coupled $T_{s}^{\mathrm{H}}$ to $T_{g}$ until $z \sim 7$ through the WF effect (without heating the gas), a similar detection might be made by a smaller experiment with $L \sim$ $2.5 \mathrm{~km}$ and $\Delta \nu_{d} \sim 1 \mathrm{kHz}$. Although it strongly enhances the 21-cm signal, the WF effect does not improve the $[\mathrm{D} / \mathrm{H}]$ measurement by the same margin once $\sigma_{\mathrm{H}_{h}}^{2} \gg$ $\sigma_{N_{h}}^{2}$ : In that regime, it only makes the $\mathrm{H}$ fluctuations a better template. Finally, we note that an experiment capable of mapping $21-\mathrm{cm}$ brightness-temperature fluctuations out to $l_{\max } \sim 10^{5}$ (where it may be a powerful probe of the small-scale matter power spectrum [4]) could measure $[\mathrm{D} / \mathrm{H}]$ to a precision as good as $\sim 1 \%$ - or even $\sim 0.1 \%$ if the WF effect coupling is efficient.

For these estimates, we have assumed a $\Lambda \mathrm{CDM}$ cosmology with $n_{s}=1$ and that a significant fraction of the Universe remains neutral until $z \sim 7$. The largest contribution to the signal originates from $z \lesssim 10$, where the D signal peaks and the variance in the density fluctuations is largest. Complete and sudden reionization at $z_{r}=10$ (14) would reduce the observable signal by a factor of 5 (20). However, a long phase of partial ionization would have much less severe effects (only reducing the number of available pixels by the filling factor of partially neutral gas). Moreover, any heating from these ionizing sources would boost the signal by strengthening the $\mathrm{H}$ template (upper curves in Fig. 3): If heating begins at $z=14$, $(\mathcal{S} / \mathcal{N})_{\text {tot }}$ decreases by only a factor of 2 relative to our fiducial case if $z_{r}=10$ - and it even increases the signal by a factor of 2 if $z_{r}=7$.

Discussion. - Despite the obvious technical challenges in observing this signal, it has the virtue of providing the cleanest possible measurement of the primordial $[\mathrm{D} / \mathrm{H}]$, free from contamination by structure formation processes at lower $z$. Via the window of BBN, this would allow radio telescopes to peer into the first few minutes of the Universe. We believe future searches for cosmic $21-\mathrm{cm}$ fluctuations should bear this possibility in mind.

We also note that ${ }^{3} \mathrm{He}^{+}$has a hyperfine transition (with $\lambda=3.46 \mathrm{~cm}$ ) that can be used in a similar fashion; it has the advantage of much lower foreground contamination at higher frequencies. This line will show a strong anticorrelation with the $21-\mathrm{cm}$ signal during reionization. If the details of reionization can be understood well enough, the cross-correlation of this line with the $21-\mathrm{cm}$ line could supplement the D-H experiment, especially if the Universe has a long period of partial ionization.

We thank A. Dalgarno, J. Gair, M. Hayden, M. Kamionkowski, T. Pearson, M. Reynolds, D. Scott, and B. Zygelman for discussions. K. S. acknowledges the support of the Canadian NSERC. This work was supported in part by NASA Grant No. NAG5-9821 and DOE Grant No. DE-FG03-92-ER40701.

*Electronic address: ksigurds@tapir.caltech.edu †Electronic address: sfurlane@tapir.caltech.edu

[1] D. N. Spergel et al., Astrophys. J. Suppl. Ser. 148, 175 (2003).

[2] E. Fermi, Z. Phys. 60, 320 (1930).

[3] C. J. Hogan and M. J. Rees, Mon. Not. R. Astron. Soc. 188, 791 (1979); D. Scott and M. J. Rees, Mon. Not. R. Astron. Soc. 247, 510 (1990).

[4] A. Loeb and M. Zaldarriaga, Phys. Rev. Lett. 92, 211301 (2004).

[5] J.E. Nafe et al., Phys. Rev. 71, 914 (1947); S. Weinreb, Nature (London) 195, 367 (1962); J. M. Pasachoff and D. A. Cesarsky, Astrophys. J. 193, 65 (1974); K. R. Anantharamaiah and V. Radhakrishnan, Astron. Astrophys. 79, L9 (1979); L. Blitz and C. Heiles, Astrophys. J. Lett. 313, L95 (1987); J. N. Chengalur et al., Astron. Astrophys. 318, L35 (1997).

[6] R. V. Wagoner et al., Astrophys. J. 148, 3 (1967).

[7] H. Reeves et al., Astrophys. J. 179, 909 (1973).

[8] C. Cohen-Tannoudji et al., Quantum Mechanics (Wiley, New York, 1977), Vol. 2.

[9] S. A. Wouthuysen, Astron. J. 57, 31 (1952).

[10] G. B. Field, Proc. IRE 46, 240 (1958).

[11] A. Dalgarno, Proc. R. Soc. A 262, 132 (1961); F. J. Smith, Planet. Space Sci. 14, 929 (1966); A.C. Allison and A. Dalgarno, Astrophys. J. 158, 423 (1969); A.C. Allison, Phys. Rev. A 5, 2695 (1972).

[12] B. Zygelman, Astrophys. J. 622, 1356 (2005).

[13] B. Shizgal, J. Phys. B 12, 3611 (1979).

[14] It has been computed at higher temperatures in Ref. [15], at $1 \mathrm{~K}$ in Ref. [16], and measured at $1 \mathrm{~K}$ in Ref. [17].

[15] P.S. Krstic and D. R. Schultz, Phys. Rev. A 60, 2118 (1999).

[16] M.W. Reynolds, Ph.D. thesis, University of British Columbia, 1989.

[17] M. E. Hayden and W. N. Hardy, J. Low Temp. Phys. 99, 787 (1995).

[18] S. Seager et al., Astrophys. J. 523, L1 (1999); Astrophys. J. Suppl. Ser. 128, 407 (2000).

[19] P.J.E. Peebles, Principles of Physical Cosmology (Princeton University Press, Princeton, 1993).

[20] S. Bharadwaj and S. S. Ali, Mon. Not. R. Astron. Soc. 352, 142 (2004).

[21] R. Barkana and A. Loeb, Astrophys. J. 624, L65 (2005).

[22] M. Zaldarriaga et al., Astrophys. J. 608, 622 (2004).

[23] M. F. Morales and J. Hewitt, Astrophys. J. 615, 7 (2004); M. G. Santos et al., Astrophys. J. 625, 575 (2005); M. McQuinn et al., astro-ph/0512263 [Astrophys. J. (to be published)]. 